\title{
Polysaccharide Conjugate Vaccine against Pneumococcal Pneumonia in Adults
}

\author{
M.J.M. Bonten, S.M. Huijts, M. Bolkenbaas, C. Webber, S. Patterson, S. Gault, \\ C.H. van Werkhoven, A.M.M. van Deursen, E.A.M. Sanders, T.J.M. Verheij, \\ M. Patton, A. McDonough, A. Moradoghli-Haftvani, H. Smith, T. Mellelieu, \\ M.W. Pride, G. Crowther, B. Schmoele-Thoma, D.A. Scott, K.U. Jansen, \\ R. Lobatto, B. Oosterman, N. Visser, E. Caspers, A. Smorenburg, E.A. Emini, \\ W.C. Gruber, and D.E. Grobbee
}

The authors' full names, academic degrees, and affiliations are listed in the Appendix. Address reprint requests to Dr. Bonten at the Julius Center for Health Sciences and Primary Care, University Medical Center Utrecht, P.O. Box 85500 Utrecht 3508 GA, the Netherlands, or at mbonten@umcutrecht.nl, or to Dr. Gruber at Pfizer Vaccine Clinical Research, 401 N. Middletown Rd., Pearl River, NY 10965, or at bill.gruber@pfizer.com.

Drs. Huijts and Bolkenbaas contributed equally to this article.

N Engl J Med 2015;372:1114-25. DOI: $10.1056 /$ NEJMoal408544

Copyright $(2015$ Massachusetts Medical Society.
ABSTRACT

\section{BACKGROUND}

Pneumococcal polysaccharide conjugate vaccines prevent pneumococcal disease in infants, but their efficacy against pneumococcal community-acquired pneumonia in adults 65 years of age or older is unknown.

\section{METHODS}

In a randomized, double-blind, placebo-controlled trial involving 84,496 adults 65 years of age or older, we evaluated the efficacy of 13-valent polysaccharide conjugate vaccine (PCV13) in preventing first episodes of vaccine-type strains of pneumococcal community-acquired pneumonia, nonbacteremic and noninvasive pneumococcal community-acquired pneumonia, and invasive pneumococcal disease. Standard laboratory methods and a serotype-specific urinary antigen detection assay were used to identify community-acquired pneumonia and invasive pneumococcal disease.

\section{RESULTS}

In the per-protocol analysis of first episodes of infections due to vaccine-type strains, community-acquired pneumonia occurred in 49 persons in the PCV13 group and 90 persons in the placebo group (vaccine efficacy, 45.6\%; $95.2 \%$ confidence interval [CI], 21.8 to 62.5), nonbacteremic and noninvasive community-acquired pneumonia occurred in 33 persons in the PCV13 group and 60 persons in the placebo group (vaccine efficacy, $45.0 \%$; $95.2 \% \mathrm{CI}, 14.2$ to 65.3 ), and invasive pneumococcal disease occurred in 7 persons in the PCV13 group and 28 persons in the placebo group (vaccine efficacy, $75.0 \%$; $95 \%$ CI, 41.4 to 90.8 ). Efficacy persisted throughout the trial (mean follow-up, 3.97 years). In the modified intention-totreat analysis, similar efficacy was observed (vaccine efficacy, 37.7\%, 41.1\%, and $75.8 \%$, respectively), and community-acquired pneumonia occurred in 747 persons in the PCV13 group and 787 persons in placebo group (vaccine efficacy, 5.1\%; 95\% $\mathrm{CI},-5.1$ to 14.2). Numbers of serious adverse events and deaths were similar in the two groups, but there were more local reactions in the PCV13 group.

CONCLUSIONS

Among older adults, PCV13 was effective in preventing vaccine-type pneumococcal, bacteremic, and nonbacteremic community-acquired pneumonia and vaccinetype invasive pneumococcal disease but not in preventing community-acquired pneumonia from any cause. (Funded by Pfizer; CAPITA ClinicalTrials.gov number NCT00744263.) 
TREPTOCOCCUS PNEUMONIAE, A MAJOR CAUSE of community-acquired pneumonia in the elderly, results in considerable morbidity and mortality. ${ }^{1-4}$ Pneumococcal community-acquired pneumonia most commonly presents as nonbacteremic disease. ${ }^{3}$ Invasive pneumococcal disease, which involves infection of normally sterile sites, occurs in approximately $25 \%$ of cases. $^{3}$

Immunologic protection against pneumococcal disease is mediated through opsonophagocytic antibodies directed against bacterial capsular polysaccharides that define the pneumococcal serotypes and serve as virulence factors. ${ }^{5}$ Vaccines composed of purified capsular polysaccharides, which have been available for more than 50 years, are not immunogenic in young children. ${ }^{6-8}$

Although some studies have shown that purified capsular polysaccharides are effective against invasive pneumococcal disease in adults, their efficacy against noninvasive pneumonia is uncertain. ${ }^{9-14}$ Pneumococcal polysaccharide conjugate vaccines (in which a bacterial polysaccharide is covalently conjugated to an immunogenic carrier protein) are effective in preventing pneumococcal pneumonia, invasive pneumococcal disease, and otitis media in children ${ }^{15-20}$ and pneumococcal infections in adults and children with human immunodeficiency virus (HIV) infection. ${ }^{17,21}$ The efficacy of pneumococcal conjugate vaccines for the prevention of vaccine-type invasive and noninvasive community-acquired pneumonia in adults 65 years of age or older has not been determined.

The assessment of efficacy became possible with the development of a serotype-specific urinary antigen detection assay. ${ }^{22,23}$ Using this assay, we were able to evaluate a 13-valent pneumococcal conjugate vaccine (PCV13) for the prevention of vaccine-type invasive and noninvasive community-acquired pneumonia in adults 65 years of age or older.

\section{METHODS}

\section{STUDY DESIGN AND OVERSIGHT}

The Community-Acquired Pneumonia Immunization Trial in Adults (CAPITA) involved a collaboration between the University Medical Center Utrecht (UMCU) and the study sponsor, Wyeth (a Pfizer company). The study design has been published previously. ${ }^{24}$ This study was a parallel-group, randomized, placebo-controlled, double-blind trial. Use of a placebo was appropriate because no pneumococcal vaccine is recommended in the Netherlands for routine use in older adults. ${ }^{25,26}$ Details of the study methods and statistical analysis plan - including randomization, objectives, definition of end points, stopping criteria, and statistical analyses - are provided in the Supplementary Appendix, available with the full text of this article at NEJM.org.

The study was conducted in compliance with Good Clinical Practice guidelines and was approved by the Central Committee on Research Involving Human Subjects and by the Ministry of Health, Welfare and Sport in the Netherlands. The UMCU and Julius Clinical, an academic research organization affiliated with the UMCU, conducted the study and gathered all the data. The UMCU and the sponsor developed the study protocol, which is available at NEJM.org. The UMCU, Julius Clinical, and the sponsor provided study oversight and managed study logistics. The sponsor performed data management, urinary antigen tests, and the statistical analysis. The first author wrote the initial draft of the manuscript. All the authors participated in writing the final version of the manuscript and vouch for the accuracy and completeness of the reported data.

\section{PARTICIPANTS}

The participants, who were 65 years of age or older, were enrolled in the study between September 15, 2008, and January 30, 2010, at 101 temporary community-based sites throughout the Netherlands. A subgroup of participants (the safety subgroup) was enrolled separately but was included in the overall study population. These participants were followed with home visits for 2 years to obtain additional safety data. Key eligibility criteria were no previous pneumococcal vaccination and an absence of protocol-defined immunocompromising conditions (see the exclusion criteria in the Supplementary Appendix). All the participants provided written informed consent.

During the first 2.5 months of study enrollment, trivalent inactivated influenza vaccine was administered concomitantly with the study vaccine or placebo in participants who were not in the safety subgroup; $30.4 \%$ of the participants received both vaccines at the vaccination visit. 
Rates of influenza vaccination in the Netherlands among people 65 years of age or older during the course of the study were reported to be 77.2 to $82.5 \% .^{27}$

The trial was completed in accordance with the protocol. All data were analyzed according to the vaccine administered. All participants who underwent randomization were vaccinated by investigators who were unaware of the study-vaccine assignments. Randomization was achieved by administration of vaccine from the next available syringe in a tray ordered in accordance with the randomization schedule. The schedule was generated with the use of a randomized block design in which study groups were randomly ordered within each block of 10 participants (i.e., each tray of 10 syringes, with six trays per shipment box). Further details are provided in the Supplementary Appendix. There were no postrandomization exclusions before vaccination.

\section{INVESTIGATIONAL PRODUCTS}

Participants were randomly assigned in a 1:1 ratio to receive PCV13 or placebo by intramuscular injection in the right deltoid. PCV13 contains polysaccharides from pneumococcal serotypes 1 , 3, 4, 5, 6A, 6B, 7F, 9V, 14, 18C, 19A, 19F, and 23F individually conjugated to a nontoxic diphtheria toxin cross-reactive material 197 protein. The vaccine contains $2.2 \mu \mathrm{g}$ of each polysaccharide (except for $4.4 \mu \mathrm{g}$ of serotype 6B), along with 5.0 $\mathrm{mM}$ succinate buffer, $0.85 \%$ sodium chloride, $0.02 \%$ polysorbate 80 , and $0.125 \mathrm{mg}$ of aluminum as aluminum phosphate per $0.5-\mathrm{ml}$ dose.

The placebo contained $5.0 \mathrm{mM}$ succinate buffer, $0.85 \%$ sodium chloride, $0.02 \%$ polysorbate 80 , and $0.125 \mathrm{mg}$ of aluminum as aluminum phosphate per $0.5-\mathrm{ml}$ dose. It was identical in appearance to PCV13.

\section{OBJECTIVES}

The primary objective was to show the efficacy of PCV13 in the prevention of a first episode of confirmed vaccine-type community-acquired pneumonia (defined as the presence of $\geq 2$ prespecified clinical criteria, findings on chest radiography consistent with community-acquired pneumonia, and a positive vaccine-type-specific urinary antigen test or isolation of vaccine-type S. pneumoniae from blood or another sterile site). The secondary objectives were to show the efficacy of PCV13 in the prevention of a first episode of confirmed nonbacteremic and noninvasive vaccine-type community-acquired pneumonia (i.e., an episode of vaccine-type community-acquired pneumonia for which the result of a blood culture and results of cultures of any other sterile site were negative for S. pneumoniae) and vaccine-type invasive pneumococcal disease (i.e., an episode in which the presence of S. pneumoniae is detected in a sterile site).

The following prespecified selected exploratory end points are also included in this article: all episodes of confirmed vaccine-type community-acquired pneumonia, a first episode of confirmed pneumococcal community-acquired pneumonia irrespective of serotype, a first episode of confirmed nonbacteremic and noninvasive pneumococcal community-acquired pneumonia irrespective of serotype, a first episode of invasive pneumococcal disease irrespective of serotype, a first episode of community-acquired pneumonia including nonpneumococcal pneumonia, and death. Analyses of all end points were prespecified in the study protocol.

The safety objective was an evaluation of the safety profile of PCV13 as measured by the incidence rates of serious adverse events for 28 days after vaccination among all participants and for 6 months after vaccination among participants in the safety subgroup. Additional safety objectives were evaluations of the frequency of local reactions, systemic events, and adverse events among participants in the safety subgroup and an assessment of the number of deaths from any cause among all participants.

\section{PROCEDURES}

Surveillance for suspected pneumonia and invasive pneumococcal disease was conducted from September 15, 2008, through August 28, 2013; the study surveillance period ended after a prespecified number of first episodes of vaccinetype community-acquired pneumonia had been identified. Surveillance was conducted at 59 sentinel centers located in the regions in which participants were enrolled.

A diagnostic routine reflecting standard of practice was used for all patients who presented to sentinel centers with symptoms of lower respiratory tract infection. If pneumonia was suspected, the staff at the sentinel center determined whether the patient was a participant in the trial by searching a participant database. If 
the patient was a study participant, an aliquot of urine was obtained for the serotype-specific urinary antigen detection, and BinaxNOW assays were performed by the sponsor (Table S7 in the Supplementary Appendix). Results of chest radiography were read centrally to provide consistent interpretation. The radiologists were unaware of the participants' vaccination status. Surveillance for invasive pneumococcal disease was conducted by regular review of laboratory culture results.

Surveillance for death, loss to follow-up, and receipt of any nonstudy pneumococcal vaccine was conducted in the same time period by means of a regular review of records from general practitioners. Physicians reviewed available documents to determine the cause of each death and to assess whether the death was due to community-acquired pneumonia or invasive pneumococcal disease.

\section{POPULATIONS FOR ANALYSIS}

The modified intention-to-treat efficacy population included participants who had an episode of community-acquired pneumonia or invasive pneumococcal disease with the onset of symptoms at least 14 days after vaccination. The modified intention-to-treat population was further categorized into two subgroups according to the participant's immune status at the time of presentation with community-acquired pneumonia or invasive pneumococcal disease: one comprising immunodeficient or immunosuppressed persons, and the other comprising immunocompetent persons. Participants who became immunodeficient or immunosuppressed after enrollment were those who met the criteria for immunodeficiency or immunosuppression specified for study eligibility (see the Supplementary Appendix) or were assessed by physicians who were experienced in clinical immunology and could determine whether the patients were immunosuppressed to the degree represented by the list of conditions in the study eligibility criteria.

The per-protocol population included participants who met the criteria for the modified intention-to-treat population, were eligible for the study, received a vaccination, and had no other major protocol violations. Episodes of communityacquired pneumonia or invasive pneumococcal disease were excluded from the per-protocol analyses if the onset of symptoms occurred after receipt of any nonstudy pneumococcal vaccine or after any of the following diagnoses: immunode- ficiency or immunosuppression (as defined in the eligibility criteria or by physician assessment that the participant was immunosuppressed), bronchial obstruction due to primary lung cancer, another cancer that was metastatic to the lungs, postobstructive pneumonia (not including chronic obstructive pulmonary disease), the acquired immunodeficiency syndrome, or known or suspected Pneumocystis jiroveci pneumonia or active tuberculosis.

\section{SAFETY ASSESSMENT}

Participants in the safety subgroup used electronic diaries to record any local reactions, systemic events, or the receipt of medications for fever or pain for 7 days after vaccination. Study nurses conducted home visits at which they collected information about other adverse events and newly diagnosed chronic medical conditions (e.g., asthma, emphysema, hypertension, and cardiac failure) in these participants. Surveillance for serious adverse events and deaths in all participants was conducted by regular review of records from general practitioners and of information reported by participants, relatives, and general practitioners.

All participants who received the study vaccine were included in the safety analysis unless safety data were lacking. Deaths, serious adverse events, and adverse events were categorized according to the Medical Dictionary for Regulatory Activities, version 16.1. All safety data were summarized according to study group.

\section{STATISTICAL ANALYSIS}

The per-protocol population was the primary population for the analysis of the primary and secondary end points and most exploratory end points. The analysis of all episodes of communityacquired pneumonia (which included nonpneumococcal pneumonia) was based on the modified intention-to-treat population.

A total of 130 primary end-point events were required to have sufficient statistical power for the final analysis; this number was determined with the use of the conditional binomial approach described by Breslow and Day, ${ }^{28}$ taking the specificity and sensitivity of the serotype-specific urinary antigen detection assay into account. The interim analysis by the data and safety monitoring committee approximately midway through the study did not result in discontinuation of the 
study. The efficacy estimates and two-sided confidence intervals were derived with the use of the Clopper-Pearson method ${ }^{29}$ with alpha adjustment for interim analysis. Study personnel remained unaware of the study-group assignments until the database was finalized.

\section{RESULTS}

\section{STUDY PARTICIPANTS}

Of the 84,496 persons who were enrolled in the study, 42,240 received PCV13 and 42,256 received placebo (Fig. 1). The mean follow-up time was 3.97 years in each group. The rates of discontinuation over the course of the study were $12.4 \%$ in the PCV13 group and $12.6 \%$ in the placebo group; discontinuations were due primarily to deaths (7.1\% in each group) and loss to follow-up (4.8\% in the PCV13 group and 5.1\% in the placebo group). Baseline characteristics were balanced between the two groups (Table 1).

\section{EFFICACY}

There were 3232 visits (by 1552 participants in the PCV13 group and 1680 in the placebo group) to sentinel centers for suspected pneumonia or invasive pneumococcal disease. A total of 2842 of these participants $(88 \%)$ had received the influenza vaccine in the previous year; the proportion was balanced between study groups $(88 \%$ in each group). Results of serotype-specific urinary antigen detection tests were available for $95 \%$ of the visits, and results from blood cultures or cultures from other sterile sites were available for $79 \%$ of the visits. These percentages were similar in the two study groups. A total of 46 participants (20 in the PCV13 group and 26 in the placebo group) were excluded from the analysis because of prior receipt of pneumococcal vaccination.

We found that the vaccine had significant efficacy in the per-protocol population against first episodes of confirmed vaccine-type communityacquired pneumonia, confirmed nonbacteremic and noninvasive vaccine-type community-acquired pneumonia, and vaccine-type invasive pneumococcal disease (Table 2). A first episode of confirmed vaccine-type community-acquired pneumonia was documented in 49 participants in the PCV13 group and 90 participants in the placebo group (vaccine efficacy, 45.6\%; 95.2\% confidence interval [CI], 21.8 to $62.5 ; \mathrm{P}<0.001)$. A first episode of confirmed nonbacteremic and noninvasive vaccine-type community-acquired pneumonia was documented in 33 participants in the PCV13 group and 60 participants in the placebo group (vaccine efficacy, $45.0 \%$; $95.2 \%$ CI, 14.2 to 65.3; $\mathrm{P}=0.007$ ), and a first episode of vaccinetype invasive pneumococcal disease was documented in 7 participants in the PCV13 group and 28 participants in the placebo group (vaccine efficacy, $75.0 \%$; $95 \%$ CI, 41.4 to $90.8 ; \mathrm{P}<0.001$ ).

We also found that the vaccine had significant efficacy with respect to the same three end points in the modified intention-to-treat population (Table 2). Tables S4, S5, and S6 in the Supplementary Appendix summarize the vaccine efficacy for these three end points in subgroups defined according to immune status. The lower vaccine efficacy with respect to confirmed vaccine-type pneumococcal community-acquired pneumonia that was observed in the modified intention-to-treat population, as compared with the vaccine efficacy in the per-protocol population, may reflect the distribution of episodes between the two study groups in the immunodeficient or immunosuppressed subgroup of participants, in whom immunocompromising conditions (such as hematologic or generalized cancers) developed or who began to receive active immunosuppressive therapy (Table S4 in the Supplementary Appendix).

For all first and subsequent episodes of confirmed vaccine-type community-acquired pneumonia, the vaccine also had significant efficacy in both the per-protocol and modified intentionto-treat populations (Table 2). Vaccine efficacy against a first episode of confirmed pneumococcal community-acquired pneumonia and against a first episode of invasive pneumococcal disease, both of which included non-vaccine-type or undetermined serotypes, was also observed. The vaccine did not have significant efficacy against a first episode of confirmed nonbacteremic and noninvasive pneumococcal community-acquired pneumonia when non-vaccine-type serotypes were included ( $\mathrm{P}=0.11$ in the per-protocol population) or against a first episode of all-cause community-acquired pneumonia, which included nonpneumococcal as well as pneumococcal pneumonia (vaccine efficacy, 5.1\%; 95\% CI, -5.1 to 14.2).

The vaccine was not shown to have significant efficacy for the prevention of death from 


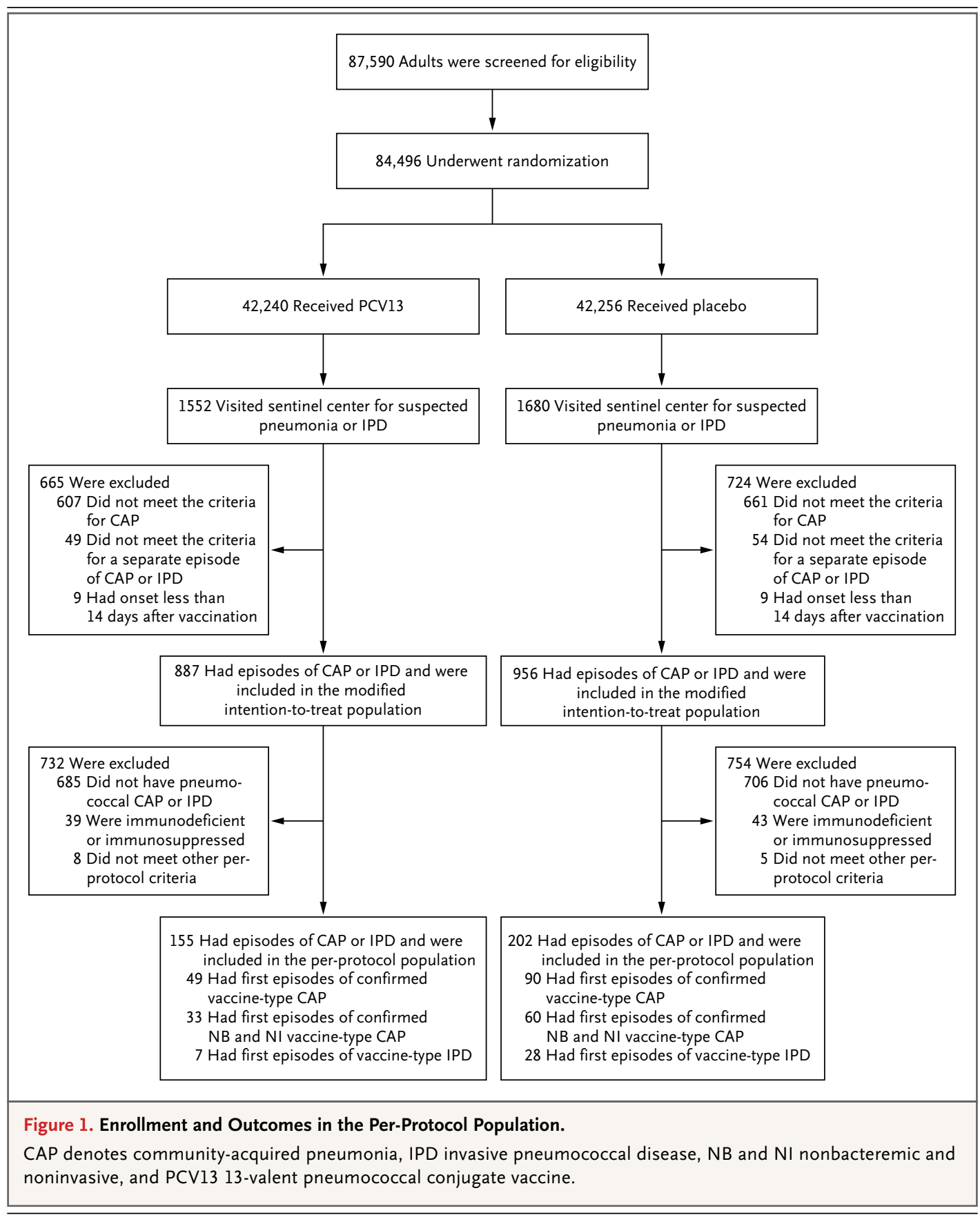

any cause. The number of deaths associated with pneumococcal disease during this study was too small to permit a meaningful analysis of the effect of the vaccine (Table S8 in the Supplementary Appendix).

Analyses of the primary and secondary end points according to serotype are summarized in Tables S10, S11, and S12 in the Supplementary Appendix. In addition, the results of the analysis of vaccine efficacy according to age against first episodes of vaccine-type community-acquired pneumonia are shown in Table S3 in the Supplementary Appendix.

Post hoc analyses of the primary and secondary end points in which the cumulative number of episodes were plotted against the time from vaccination provided evidence of efficacy beginning shortly after vaccination. They also showed 


\begin{tabular}{|c|c|c|c|}
\hline Characteristic & $\begin{array}{l}\text { PCV13 Group } \\
(\mathrm{N}=42,237) \dagger\end{array}$ & $\begin{array}{c}\text { Placebo Group } \\
(\mathrm{N}=42,255){ }^{\prime}\end{array}$ & $\begin{array}{l}\text { All Participants } \\
(\mathrm{N}=84,492){ }^{\circ}\end{array}$ \\
\hline \multicolumn{4}{|l|}{ Sex-no. (\%) } \\
\hline Male & $23,447(55.5)$ & $23,801(56.3)$ & $47,248(55.9)$ \\
\hline Female & $18,790(44.5)$ & $18,454(43.7)$ & $37,244(44.1)$ \\
\hline \multicolumn{4}{|l|}{ Race - no. (\%) $\ddagger$} \\
\hline White & $41,600(98.5)$ & $41,614(98.5)$ & $83,214(98.5)$ \\
\hline Black & $146(0.3)$ & $140(0.3)$ & $286(0.3)$ \\
\hline Asian & $277(0.7)$ & $292(0.7)$ & $569(0.7)$ \\
\hline Other & $205(0.5)$ & $199(0.5)$ & $404(0.5)$ \\
\hline Unknown & $9(<0.1)$ & $10(<0.1)$ & $19(<0.1)$ \\
\hline \multicolumn{4}{|c|}{ Age at vaccination $-y r$} \\
\hline Mean & $72.8 \pm 5.7$ & $72.8 \pm 5.6$ & $72.8 \pm 5.7$ \\
\hline Median (range) $\mathbb{S}$ & 71.6 (61.9-101.1) & 71.5 (63.3-99.5) & 71.6 (61.9-101.1) \\
\hline \multicolumn{4}{|l|}{ Age group — no. (\%) } \\
\hline$<75 \mathrm{yr}$ & $29,006(68.7)$ & $29,064(68.8)$ & $58,070(68.7)$ \\
\hline$\geq 75$ and $<85 \mathrm{yr}$ & $11,727(27.8)$ & $11,753(27.8)$ & $23,480(27.8)$ \\
\hline$\geq 85 \mathrm{yr}$ & $1504(3.6)$ & $1438(3.4)$ & $2942(3.5)$ \\
\hline \multicolumn{4}{|c|}{$\begin{array}{l}\text { * Plus-minus values are means } \pm \text { SD. Additional characteristics are listed in Table S2 in the Supplementary Appendix. } \\
\text { PCV13 denotes } 13 \text {-valent pneumococcal conjugate vaccine. } \\
\text { The numbers of participants who received the study vaccine and for whom any safety data were available are shown. } \\
\text { Four participants (three in the PCV13 group and one in the placebo group) were excluded because no safety data were } \\
\text { available. } \\
\text { Race was self-reported. } \\
\text { I A total of } 18 \text { participants who were enrolled in the PCV13 group and } 16 \text { who were enrolled in the placebo group were } \\
\text { younger than } 65 \text { years of age. }\end{array}$} \\
\hline
\end{tabular}

the persistence of efficacy throughout the duration of the trial (mean follow-up, approximately 4 years) without evidence of waning (Fig. 2).

\section{SAFETY}

The frequencies of prespecified local reactions and systemic events (Tables S13 and S14, respectively, in the Supplementary Appendix) reported by participants in the safety subgroup were higher in the PCV13 group than in the placebo group. Most local reactions and systemic events were mild or moderate in severity.

There was no significant difference between the two groups in the frequencies of newly diagnosed chronic medical conditions, serious adverse events, or deaths (Table 3). The significant difference in the percentage of participants who reported an adverse event reflects differences between the groups with respect to the prevalence of injection-site reactions and of muscular pain. No vaccine-related serious adverse events were reported.

\section{DISCUSSION}

In this study, we found that PCV13 had significant efficacy for the prevention of vaccine-type community-acquired pneumonia and vaccine-type invasive pneumococcal disease among adults 65 years of age or older, and the efficacy persisted for at least 4 years. In the per-protocol population, vaccine efficacy was $46 \%$ for the prevention of a first episode of vaccine-type communityacquired pneumonia, $45 \%$ for the prevention of nonbacteremic and noninvasive vaccine-type community-acquired pneumonia, and $75 \%$ for the prevention of vaccine-type invasive pneumococcal disease.

PCV13 had significant efficacy with respect to all three end points in the modified intentionto-treat population but not with respect to community-acquired pneumonia from any cause. Given that approximately $13 \%$ of the first episodes of community-acquired pneumonia observed in the placebo group were episodes of vaccine-type 
Table 2. Vaccine Efficacy.*

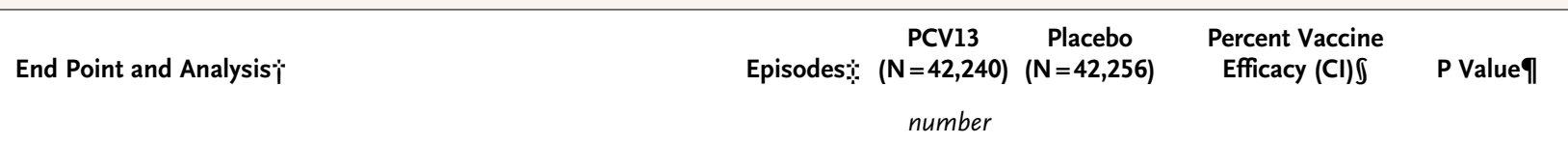

\section{First episode}

Infection with vaccine-type strain

Confirmed community-acquired pneumonia

Per-protocol analysis

Modified intention-to-treat analysis

139

172

Confirmed nonbacteremic and noninvasive communityacquired pneumonia

Per-protocol analysis

Modified intention-to-treat analysis

Invasive pneumococcal disease

Per-protocol analysis

Modified intention-to-treat analysis

Infection with any pneumococcal strain

Confirmed pneumococcal community-acquired pneumonia

Per-protocol analysis

Modified intention-to-treat analysis

Confirmed nonbacteremic and noninvasive pneumococcal community-acquired pneumonia

Per-protocol analysis

Modified intention-to-treat analysis

Invasive pneumococcal disease

Per-protocol analysis

Modified intention-to-treat analysis

Community-acquired pneumonia

Modified intention-to-treat analysis

All episodes of confirmed vaccine-type community-acquired pneumonia

Per-protocol analysis

Modified intention-to-treat analysis

Death\|

From confirmed vaccine-type pneumococcal community-acquired pneumonia or vaccine-type invasive pneumococcal disease

From confirmed pneumococcal community-acquired pneumonia or invasive pneumococcal disease

* The numbers of participants who received the vaccine in each study group are shown. $\mathrm{Cl}$ denotes confidence interval.

$\uparrow$ The modified intention-to-treat population included participants who had an episode of community-acquired pneumonia or invasive pneumococcal disease, with the onset of symptoms at least 14 days after administration of either PCV13 or placebo. Episodes of community-acquired pneumonia or invasive pneumococcal disease were excluded from per-protocol analyses if the onset of symptoms occurred after the participant received any nonstudy pneumococcal vaccine or after the participant received any of the following diagnoses: immunodeficiency or immunosuppression, bronchial obstruction due to primary lung cancer, another cancer with metastasis to the lungs, postobstructive pneumonia (not including chronic obstructive pulmonary disease), the acquired immunodeficiency syndrome, or known or suspected Pneumocystis jiroveci pneumonia or active tuberculosis.

$†$ Some participants may have had more than one episode.

$\int$ The definition of vaccine efficacy is provided in the Supplementary Appendix. Confidence intervals were derived with the use of the ClopperPearson method. The confidence intervals for the end points of confirmed vaccine-type community-acquired pneumonia and confirmed nonbacteremic and noninvasive vaccine-type community-acquired pneumonia were adjusted to $95.2 \%$ to account for the assessment of these two end points at the interim and final analyses; $95 \%$ confidence intervals are shown for the other end points.

I $\mathrm{P}$ values for exploratory end points were adjusted for multiple comparisons with the use of the Benjamini-Hochberg false-discovery-rate procedure within each population group.

\| The number of deaths shown are those that were determined by the mortality assessment committee to be due to community-acquired pneumonia or invasive pneumococcal disease and for which there was an episode detected by the sentinel system with a date of resolution of symptoms of the episode within 7 days before death. These deaths were related to episodes of community-acquired pneumonia and invasive pneumococcal disease in participants who were members of the per-protocol case-level population (i.e., participants with episodes that met all of the criteria for community-acquired pneumonia). 


\begin{tabular}{|c|c|c|c|c|c|c|}
\hline \multirow[t]{3}{*}{ Event } & \multicolumn{2}{|c|}{ Safety Subgroup } & \multirow[t]{2}{*}{ P Value'̀ } & \multicolumn{2}{|c|}{ All Participants } & \multirow[t]{3}{*}{ P Value } \\
\hline & $\begin{array}{c}\mathrm{PCV} 13 \\
(\mathrm{~N}=1006)\end{array}$ & $\begin{array}{l}\text { Placebo } \\
(\mathrm{N}=1005)\end{array}$ & & $\begin{array}{c}\mathrm{PCV} 13 \\
(\mathrm{~N}=42,237)\end{array}$ & $\begin{array}{c}\text { Placebo } \\
(\mathrm{N}=42,255)\end{array}$ & \\
\hline & \multicolumn{2}{|c|}{ no. (\%) } & \multicolumn{3}{|c|}{ no. (\%) } & \\
\hline Adverse event within 1 mo after vaccination & $188(18.7)$ & $144(14.3)$ & 0.01 & & & \\
\hline $\begin{array}{l}\text { Chronic medical condition diagnosed l-6 mo } \\
\text { after vaccinationt }\end{array}$ & $17(1.7)$ & $12(1.2)$ & 0.46 & & & \\
\hline \multicolumn{7}{|l|}{ Serious adverse event } \\
\hline Within 6 mo after vaccination & $70(7.0)$ & $60(6.0)$ & 0.41 & & & \\
\hline Within 1 mo after vaccination & & & & $327(0.8)$ & $314(0.7)$ & 0.61 \\
\hline Death & & & & $3006(7.1)$ & $3005(7.1)$ & 0.98 \\
\hline \multicolumn{7}{|c|}{$\begin{array}{l}\text { The numbers of participants who received study vaccine and for whom any safety data were available are shown. Four participants (three in } \\
\text { the PCV13 group and one in the placebo group) were excluded because they had no safety data. Listed are events that occurred at least } \\
\text { once in any participant. } \\
\text { A two-sided Fisher's exact test was used to calculate the P value for the difference between percentages of participants who reported an } \\
\text { event in each of the study groups. } \\
\text { pewly diagnosed chronic medical conditions (including autoimmune or neuroinflammatory disease) were identified in subgroup partici- } \\
\text { pants by site staff members who conducted home visits; chronic medical conditions included conditions such as asthma, emphysema, hy- }\end{array}$} \\
\hline
\end{tabular}

pneumococcal community-acquired pneumonia, the nonsignificant $5.1 \%$ vaccine efficacy observed against a first episode of community-acquired pneumonia from any cause was consistent with the observed $46 \%$ reduction in vaccine-type pneumococcal community-acquired pneumonia.

This trial also did not identify any safety concerns associated with the use of PCV13 in more than 42,000 patients who were vaccinated. These findings are consistent with observations from previous studies of PCV13 vaccination in adults. ${ }^{30-34}$

Post hoc analyses provided evidence of efficacy beginning shortly after vaccination. This efficacy was sustained throughout the duration of the trial (mean follow-up, approximately 4 years) without evidence of waning. The immunologic basis for this persistent efficacy against both vaccine-type community-acquired pneumonia and vaccine-type invasive pneumococcal disease requires further study but may reflect both the quantity and quality of the elicited functional antibacterial immune response, as well as establishment of immune memory mediated by PCV13. . $^{30-32,35}$

Our study showed the efficacy of PCV13 for the prevention of vaccine-type nonbacteremic and noninvasive pneumococcal community-acquired pneumonia in older adults. This evaluation was made possible by the serotype-specific urinary antigen detection assay, which detects vaccinetype $S$. pneumoniae polysaccharides in the urine of persons with suspected pneumonia. Previously, diagnosis was dependent on assays that relied on isolation of the organism (culture) or that had insufficient sensitivity and specificity (such as BinaxNOW). ${ }^{36}$ Most earlier randomized studies of vaccination with either free polysaccharide vaccines in older adults ${ }^{37}$ or pneumococcal conjugate vaccines in HIV-infected adults ${ }^{21}$ were underpowered to show efficacy against vaccinetype community-acquired pneumonia; results of these studies and other observational studies have been inconsistent. ${ }^{11-14}$

Our study had several limitations. The study was performed in a single country in a homogeneous population of participants among whom there was a low incidence of pneumococcal disease. ${ }^{24}$ The degree of vaccine efficacy observed may vary in other populations depending on the epidemiologic characteristics of the vaccine-associated serotypes and the susceptibility of the population to pneumococcal infections and disease. The 7-valent pneumococcal conjugate vaccine (PCV7) was introduced in the Netherlands in 2006 for use in all newborns and was replaced by the 10-valent pneumococcal conjugate vaccine (PCV10) in March 2011. In 2008, a total of 68.4\% 


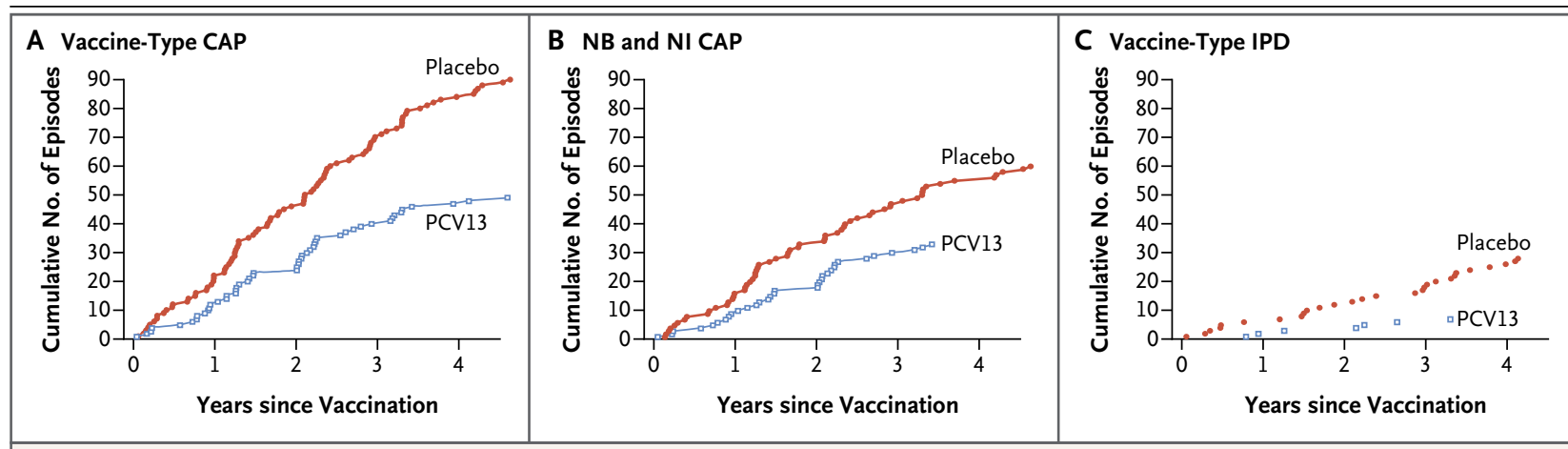

Figure 2. Post Hoc Analysis of the Cumulative Episodes of the Primary and Secondary Efficacy End Points in the Per-Protocol Population.

of the episodes of invasive pneumococcal disease among patients 65 years of age or older were caused by PCV13 serotypes $(49.7 \%$ by PCV7 serotypes and $5.3 \%$ by serotype $19 \mathrm{~A}) .^{38}$ During the study period, in 2013, this percentage decreased to $42.3 \%$ caused by PCV13 serotypes (6\% by PCV7 serotypes and $11.4 \%$ by serotype $19 \mathrm{~A})$.

In addition, the study was not designed to show efficacy among patients in whom an immunosuppressing condition developed after enrollment. Such events that occurred after randomization were excluded from the per-protocol analysis. These episodes were included as part of the analyses based on the modified intention-to-treat population; however, the numbers of immunosuppressed participants were too low to draw a meaningful conclusion regarding efficacy in this subpopulation.

Finally, the higher sensitivity of the serotypespecific urinary antigen detection assay to detect vaccine-type $S$. pneumoniae may have led to marginal overestimation of the proportion of vaccine-type serotypes and, therefore, the efficacy of the vaccine with regard to all pneumococcal community-acquired pneumonia. Nonetheless, the $46 \%$ reduction in vaccine-type communityacquired pneumonia that was seen after PCV13 immunization indicates that PCV13 immunization in adults may contribute to a reduction in pneumococcal pneumonia in older adults.

Supported by Pfizer.

Drs. Bonten and van Werkhoven and Ms. McDonough report receiving consulting fees from Pfizer; Dr. Webber, Dr. Patterson, Ms. Gault, Mr. Patton, Ms. Moradoghli-Haftvani, Ms. Smith, Ms. Mellelieu, Dr. Pride, Dr. Crowther, Dr. SchmoeleThoma, Dr. Scott, Dr. Jansen, Dr. Emini, and Dr. Gruber, being employees of and owning stock in Pfizer; Dr. Sanders, receiving grant support through her institution and fees for serving on independent data and safety monitoring committees from Pfizer and GlaxoSmithKline; and Dr. Grobbee, receiving speaking and consulting fees, honoraria, and research funding from and serving as an investigator for Pfizer, and receiving grant support from Roche Pharmaceuticals and Novartis. No other potential conflict of interest relevant to this article was reported.

Disclosure forms provided by the authors are available with the full text of this article at NEJM.org.

We thank all the study participants and the investigators; Dr. Reinier Veenhoven for substantial contributions to the enrollment of participants in the safety subgroup and collection of data in this subgroup; Drs. Keri Clarke, Dan Creswell, Wayne Drews, Jeff Goodrich, James Trammel, and Brian Tucker for contributions to the analysis and reporting of the study; and past and present members of the data and safety monitoring committee (Drs. Adam Hill, Ruth Karron, Kathleen Neuzil, Steve Self [chair], Jos van der Meer, Mark Woodhead, and Richard Wunderink).

This article is dedicated to the memory of Dr. Robert Kohberger, who contributed to the planning of the design and statistical analysis of this trial; Dr. Reinier Veenhoven, who contributed to the planning of the design and execution of the subgroup study; and Dr. Marcel Peeters, who served as a researcher in two of the sentinel hospitals.

\section{APPENDIX}

The authors' full names and academic degrees are as follows: Marc J.M. Bonten, M.D., Ph.D., Susanne M. Huijts, M.D., Marieke Bolkenbaas, M.D., Chris Webber, M.D., Scott Patterson, Ph.D., Samantha Gault, M.B.A., Cornelis H. van Werkhoven, M.D., Anna M.M. van Deursen, M.D., Elisabeth A.M. Sanders, M.D., Ph.D., Theo J.M. Verheij, M.D., Ph.D., Michael Patton, B.Sc., Anne McDonough, M.P.H., Anita Moradoghli-Haftvani, B.Sc., Helen Smith, B.Sc., Tracey Mellelieu, B.Sc., Michael W. Pride, Ph.D., Graham Crowther, Ph.D., Beate Schmoele-Thoma, M.D., Daniel A. Scott, M.D., Kathrin U. Jansen, Ph.D., Rita Lobatto, M.D., Bas Oosterman, Ph.D., Nils Visser, M.Sc., Esther Caspers, M.Sc., Andre Smorenburg, M.Sc., Emilio A. Emini, Ph.D., William C. Gruber, M.D., and Diederick E. Grobbee, M.D., Ph.D.

The authors' affiliations are as follows: the Julius Center for Health Sciences and Primary Care (M.J.M.B., S.M.H., M.B., C.H.W., A.M.M.D., T.J.M.V.) and the Department of Medical Microbiology (M.J.M.B., S.M.H., M.B., C.H.W., A.M.M.D., T.J.M.V.), University Medical Center (UMC) Utrecht, and the Department of Immunology and Infectious Diseases, Wilhelmina Children's Hospital, UMC Utrecht (A.M.M.D., E.A.M.S.), Utrecht, Research Center Linnaeus Institute, Spaarne Hospital, Hoofddorp (A.M.M.D.), and Julius 
Clinical, Zeist (R.L., B.O., N.V., E.C., A.S., D.E.G.) - all in the Netherlands; Pfizer Vaccine Clinical Research, Maidenhead, United Kingdom (C.W., S.G., M.P., A.M., A.M.-H., H.S., T.M., G.C.); Pfizer Vaccine Clinical Research, Collegeville, PA (S.P., E.A.E.); Pfizer Vaccine Research and Early Development (M.W.P., K.U.J.) and Pfizer Vaccine Clinical Research (D.A.S., E.A.E., W.C.G.), Pearl River, NY; and Pfizer Vaccine Clinical Research, Berlin (B.S.-T.).

\section{REFERENCES}

1. Peto L, Nadjm B, Horby P, et al. The bacterial aetiology of adult communityacquired pneumonia in Asia: a systematic review. Trans R Soc Trop Med Hyg 2014; 108:326-37.

2. Rozenbaum $\mathrm{MH}$, Pechlivanoglou $\mathrm{P}$, van der Werf TS, Lo-Ten-Foe JR, Postma MJ, Hak E. The role of Streptococcus pneumoniae in community-acquired pneumonia among adults in Europe: a meta-analysis. Eur J Clin Microbiol Infect Dis 2013; 32:305-16.

3. Said MA, Johnson HL, Nonyane BA, et al. Estimating the burden of pneumococcal pneumonia among adults: a systematic review and meta-analysis of diagnostic techniques. PLoS One 2013;8(4): e60273.

4. Welte T, Torres A, Nathwani D. Clinical and economic burden of communityacquired pneumonia among adults in Europe. Thorax 2012;67:71-9.

5. Kim JO, Romero-Steiner S, Sørensen UB, et al. Relationship between cell surface carbohydrates and intrastrain variation on opsonophagocytosis of Streptococcus pneumoniae. Infect Immun 1999;67: 2327-33.

6. Leinonen M, Säkkinen A, Kalliokoski $\mathrm{R}$, Luotonen J, Timonen $\mathrm{M}$, Mäkelä $\mathrm{PH}$. Antibody response to 14-valent pneumococcal capsular polysaccharide vaccine in pre-school age children. Pediatr Infect Dis 1986;5:39-44.

7. Koskela M, Leinonen M, Häivä VM, Timonen M, Mäkelä PH. First and second dose antibody responses to pneumococcal polysaccharide vaccine in infants. Pediatr Infect Dis 1986;5:45-50.

8. Baltimore RS. New challenges in the development of a conjugate pneumococcal vaccine. JAMA 1992;268:3366-7.

9. Jackson LA, Neuzil KM, Yu O, et al. Effectiveness of pneumococcal polysaccharide vaccine in older adults. $\mathrm{N}$ Engl J Med 2003;348:1747-55.

10. Honkanen PO, Keistinen T, Miettinen $\mathrm{L}$, et al. Incremental effectiveness of pneumococcal vaccine on simultaneously administered influenza vaccine in preventing pneumonia and pneumococcal pneumonia among persons aged 65 years or older. Vaccine 1999;17:2493-500.

11. Huss A, Scott P, Stuck AE, Trotter C, Egger M. Efficacy of pneumococcal vaccination in adults: a meta-analysis. CMAJ 2009;180:48-58.

12. Mangtani P, Cutts F, Hall AJ. Efficacy of polysaccharide pneumococcal vaccine in adults in more developed countries: the state of the evidence. Lancet Infect Dis 2003;3:71-8.
13. Moberley S, Holden J, Tatham DP, Andrews RM. Vaccines for preventing pneumococcal infection in adults. Cochrane Database Syst Rev 2013;1:CD000422.

14. Conaty S, Watson L, Dinnes J, Waugh $\mathrm{N}$. The effectiveness of pneumococca polysaccharide vaccines in adults: a systematic review of observational studies and comparison with results from randomised controlled trials. Vaccine 2004; 22:3214-24

15. Black S, Shinefield H, Fireman B, et al. Efficacy, safety and immunogenicity of heptavalent pneumococcal conjugate vaccine in children. Pediatr Infect Dis J 2000 19:187-95.

16. Cutts FT, Zaman SM, Enwere G, et al Efficacy of nine-valent pneumococca conjugate vaccine against pneumonia and invasive pneumococcal disease in The Gambia: randomised, double-blind, placebo-controlled trial. Lancet 2005;365: 1139-46.

17. Klugman KP, Madhi SA, Huebner RE, Kohberger R, Mbelle N, Pierce N. A tria of a 9-valent pneumococcal conjugate vaccine in children with and those without HIV infection. N Engl J Med 2003;349: 1341-8.

18. Shinefield HR, Black S. Efficacy of pneumococcal conjugate vaccines in large scale field trials. Pediatr Infect Dis J 2000 19:394-7.

19. Eskola J, Kilpi T, Palmu A, et al. Efficacy of a pneumococcal conjugate vaccine against acute otitis media. $\mathrm{N}$ Engl J Med 2001;344:403-9.

20. O'Brien KL, Moulton LH, Reid R, et al. Efficacy and safety of seven-valent conjugate pneumococcal vaccine in American Indian children: group randomised trial. Lancet 2003;362:355-61.

21. French N, Gordon SB, Mwalukomo T et al. A trial of a 7-valent pneumococca conjugate vaccine in HIV-infected adults. N Engl J Med 2010;362:812-22.

22. Pride MW, Huijts SM, Wu K, et al. Validation of an immunodiagnostic assay for detection of 13 Streptococcus pneumoniae serotype-specific polysaccharides in human urine. Clin Vaccine Immunol 2012; 19:1131-41

23. Huijts SM, Pride MW, Vos JM, et al. Diagnostic accuracy of a serotype-specific antigen test in community-acquired pneumonia. Eur Respir J 2013;42:1283 90.

24. Hak E, Grobbee DE, Sanders EAM, et al. Rationale and design of CAPITA: a RCT of 13-valent conjugated pneumococcal vaccine efficacy among older adults. Neth J Med 2008;66:378-83.
25. Health Council of the Netherlands. Pneumococcal vaccine in elderly adults and risk group. The Hague: Health Council of the Netherlands, 2003; publication no. 2003/10 (http://www.gr.nl/en/ publications/preventie/pneumococcal -vaccine-in-elderly-adults-and-risk -groups).

26. Health Council of the Netherlands. The future of the National Immunisation Programme: towards a programme for all age groups. The Hague: Health Council of the Netherlands, 2007; publication no. 2007/02 (http://www.gr.nl/en/publications/ preventie/the-future-of-the-national -immunisation-programme-towards -a-programme-for-all)

27. Jansen B, Tacken M, Mulder J, et al. Monitoring vaccination rate Dutch $\mathrm{Na}$ tional Influenza Prevention Programme 2011. Nijmegen: Scientific Institute for Quality of Healthcare (IQ healthcare), 2012 (http://www.snpg.nl/producten/2012/ Monitoring-vaccinatiegraad-NPG-2011 .pdf [Dutch version] or http://www .iqhealthcare.nl/media/9715/verkorte_ versie_rapport_griep.pdf [Dutch and English version]).

28. Breslow NE, Day NE. Statistical methods in cancer research: vol. 1: the analysis of case-control studies. Lyon, France: International Agency for Research on Cancer, 1980 (http://www.iarc.fr/en/ publications/pdfs-online/stat/sp32/ SP32_vol1-0.pdf).

29. Clopper CJ, Pearson ES. The use of confidence or fiducial limits illustrated in the case of the binomial. Biometrika 1934;26:403-14.

30. Jackson LA, Gurtman A, van Cleeff M, et al. Immunogenicity and safety of a 13-valent pneumococcal conjugate vaccine compared to a 23-valent pneumococcal polysaccharide vaccine in pneumococcal vaccine-naive adults. Vaccine 2013;31: 3577-84.

31. Jackson LA, Gurtman A, Rice K, et al. Immunogenicity and safety of a 13-valent pneumococcal conjugate vaccine in adults 70 years of age and older previously vaccinated with 23-valent pneumococcal polysaccharide vaccine. Vaccine 2013;31: 3585-93.

32. Greenberg RN, Gurtman A, Frenck $\mathrm{RW}$, et al. Sequential administration of 13-valent pneumococcal conjugate vaccine and 23-valent pneumococcal polysaccharide vaccine in pneumococcal vaccinenaïve adults $60-64$ years of age. Vaccine 2014;32:2364-74.

33. Schwarz TF, Pauksens K, Juergens C, et al. Safety of a 13 -valent pneumococcal 
conjugate vaccine in elderly adults previously immunized with a 23-valent pneumococcal polysaccharide vaccine: an open-label trial. World J Vaccines 2013;3 123-9.

34. Schwarz TF, Flamaing J, Rümke HC, et al. A randomized, double-blind trial to evaluate immunogenicity and safety of 13-valent pneumococcal conjugate vaccine given concomitantly with trivalent influenza vaccine in adults aged $\geq 65$ years. Vaccine 2011;29:5195-202.

35. Jackson LA, Gurtman A, van Cleeff M, et al. Influence of initial vaccination with 13-valent pneumococcal conjugate vaccine or 23-valent pneumococcal polysaccharide vaccine on anti-pneumococcal responses following subsequent pneumococcal vaccination in adults 50 years and older. Vaccine 2013;31:3594-602.

36. Sherwin RL, Gray S, Alexander R, et al. Distribution of 13-valent pneumococcal conjugate vaccine Streptococcus pneumoniae serotypes in US adults aged $\geq 50$ years with community-acquired pneumonia. J Infect Dis 2013;208:1813-20.
37. Fedson DS, Liss C. Precise answers to the wrong question: prospective clinical trials and the meta-analyses of pneumococcal vaccine in elderly and high-risk adults. Vaccine 2004;22:927-46.

38. van Deursen AM, van Mens SP, Sanders EA, et al. Invasive pneumococcal disease and 7-valent pneumococcal conjugate vaccine, the Netherlands. Emerg Infect Dis 2012;18:1729-37.

Coppright $(2015$ Massachusetts Medical Society. 